\title{
On Locality of Dominating Set in Ad Hoc Networks with Switch-On/Off Operations *
}

\author{
Jie Wu and Fei Dai \\ Department of Computer Science and Engineering \\ Florida Atlantic University \\ Boca Raton, FL 33431
}

\begin{abstract}
Routing based on a connected dominating set is a promising approach, where the search space for a route is reduced to the hosts in the set. A set is dominating if all the hosts in the system are either in the set or neighbors of hosts in the set. In this paper, we first review a distributed formation of a connected dominating set called marking process and dominating-set-based routing. Then we propose several ways to reduce the size of the dominating set and study the locality of dominating set in ad hoc wireless networks with switch-on/off operations. Results show that the dominating set derived from the marking process exhibits good locality properties; i.e., the change of a host status, gateway (dominating) or non-gateway (dominated), affects only the status of hosts in a restricted vicinity.
\end{abstract}

\section{Introduction}

Dominating-set-based routing [8] is a promising routing approach in ad hoc networks. A subset of the vertices of a graph is a dominating set if every vertex not in the subset is adjacent to at least one vertex in the subset. Moreover, this dominating set should be connected for ease of the routing process within the induced graph defined to consist of dominating vertices only. Vertices in a dominating set are also called gateway hosts while vertices that are outside a dominating set are called non-gateway hosts. We can use a unit disk graph [2] $G=(V, E)$ to represent an ad hoc network, where $V$ represents a set of wireless mobile hosts and $E$ represents a set of edges. An edge between a host pair $(u, v)$ indicates that both hosts $u$ and $v$ are within their wireless transmitter ranges. In Figure 1 (b), hosts $u$ and $v$ form a connected dominating set of the given unit disk graph.

The main advantage of dominating-set-based routing is that it simplifies the routing process to the one in a smaller

* This work was supported in part by NSF grant CCR 9900646 and grant ANI 0073736. Email: \{jie, fdai\}@cse.fau.edu. subnetwork generated from the connected dominating set. This means that only gateway hosts need to keep routing information in a proactive approach and the search space is reduced to the dominating set in a reactive approach. In proactive routing, routes to all destinations are computed $a$ priori and are maintained in the background via a periodic update process. In reactive routing, a route to a specific destination is computed "on demand"; i.e., only when needed. Clearly, the efficiency of this approach depends largely on the process of finding and maintaining a connected dominating set and the size of the corresponding subnetwork.

Unfortunately, finding a minimum connected dominating set is NP-complete for most graphs. Wu and Li [8] proposed a simple and efficient distributed algorithm that can quickly determine a connected dominating set in ad hoc networks. This approach uses a localized algorithm called marking process where hosts interact with others in the neighborhood. Specifically, each host is marked true if it has two unconnected neighbors. It is shown that collectively these hosts achieve a desired global objective - a set of marked hosts forms a small connected dominating set.

In this paper, we focus on maintaining the dominating set in an ad hoc network where switch-on/off operations are major operations that change network topology. Such a network can be either a sensor network [3] with limited mobility or a rooftop network [6] without mobility, but is deployed very densely in metropolitan areas. We study the locality of dominating set in ad hoc networks with switchon/off operations. The dominating set under consideration is derived from the marking process and it is further reduced through different reduction methods proposed in this paper. The main contributions of the paper include the locality property of the marking process. That is, the change of a host status, gateway (dominating) or non-gateway (dominated), affects only the status of hosts in a restricted vicinity. In addition, locality of host status update is also verified through simulation. We show the different locality properties of gateway/non-gateway derived by different versions of the marking process. 


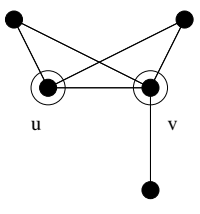

(a)

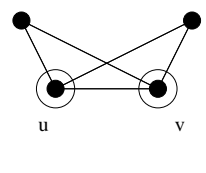

(b)

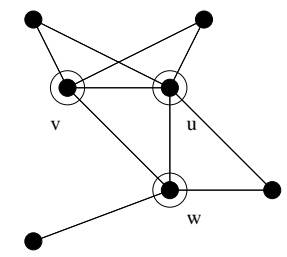

(c)
Figure 1. Dominating set reduction examples.

\section{Preliminaries}

We first review the marking process [8]: (1) Initially assign marker $F$ to each $v$ in $V$. (2) Each $v$ exchanges its neighbor set $N(v)$ with all its neighbors. (3) Each $v$ assigns its marker $m(v)$ to $T$ if there exist two unconnected neighbors.

The marking process is a localized algorithm, where hosts only interact with others in the neighborhood. Suppose $m(v)$ is a marker for vertex $v \in V$, which is either $T$ (marked) or $F$ (unmarked). Each vertex $v$ always maintains its neighbor set $N(v)=\{u \mid(v, u) \in E\}$. Assume that $V^{\prime}$ is the set of vertices that are marked $T$ in $V$; i.e., $V^{\prime}=\{v \mid v \in V, m(v)=T\}$. The induced graph $G^{\prime}$ is the subgraph of $G$ induced by $V^{\prime}$; i.e., $G^{\prime}=G\left[V^{\prime}\right]$. The following results [8] show that $V^{\prime}$ is a connected dominating set of $G$.

Property 1 Given a connected graph $G$ that is not completely connected, the vertex subset $V^{\prime}$, derived from the marking process, forms a connected dominating set of $G$.

Property 2 The shortest path between any two vertices does not include any non-gateway vertex as an intermediate host.

Since the problem of determining a minimum connected dominating set of a given connected graph is NP-complete, the connected dominating set derived from the marking process is normally non-minimum. In some cases, the resultant dominating set is trivial; i.e., $V^{\prime}=V$ or $V^{\prime}=\{\}$. For example, any vertex-symmetric graph will generate a trivial dominating set using the proposed marking process. However, the marking process is efficient for ad hoc networks where the corresponding unit disk graph tends to form a set of localized clusters (or cliques).

Dominating-set-based routing usually consists three steps: (1) If the source is not a gateway host, it forwards the packets to a source gateway, which is one of the adjacent gateway hosts. (2) This source gateway acts as a new source to route the packets in the induced graph generated from the connected dominating set. (3) Eventually, the packets reach a destination gateway, which is either the destination host

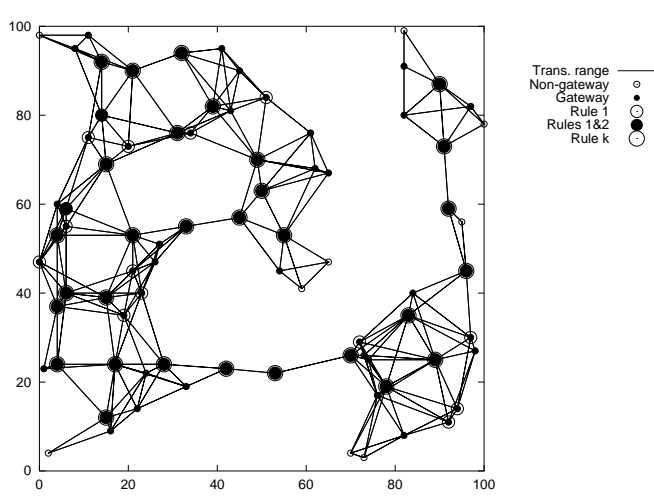

Figure 2. A sample ad hoc network.

itself or a gateway that connects the destination host. In the later case, the destination gateway forwards the packets directly to the destination host.

There are in general two ways to perform routing within the induced graph: proactive routing and reactive routing. In [8], DSDV [5] is used as a sample proactive routing to illustrate the dominating-set-based routing. Using the $n s-2$ simulator, Sinha, Sivalumar, and Bharghavan [7] evaluate the performance of DSR [1] and AODV [4] (both are reactive routing), when they are operated over the dominating set (called core in [7]) and compare their performance against those of their basic versions.

\section{Dominating Set Reduction}

In this section, we propose several ways (in form of rules) to reduce the size of the connected dominating set derived from the marking process. We first assign a distinct id, $i d(v)$, to each vertex $v$ in $V^{\prime}$.

Rule 1: Consider two vertices $u$ and $v$ in $G^{\prime}$. If $N(u)-$ $\{v\} \subseteq N(v)$ in $G$ and $i d(u)<i d(v)$, change the marker of $u$ to $F$; i.e., $V^{\prime}$ is changed to $V^{\prime}-\{u\}$.

It is easy to prove that $V^{\prime}-\{u\}$ is still a connected dominating set of $G$. In addition, Property 2 still holds. Note that $u$ and $v$ in Rule 1 may or may not be neighbors.

In Figure 1 (a), since $N(u)-\{v\} \subseteq N(v)$, vertex $u$ is removed from $V^{\prime}$ if $i d(u)<i d(v)$ and vertex $v$ is the only dominating vertex in the graph. In Figure 1 (b), since $u$ and $v$ cover each other, either $u$ or $v$ can be removed from $V^{\prime}$. To ensure one and only one is removed, we pick the one with a smaller id.

Rule 2: Assume that $v$ and $w$ are neighbors in $G^{\prime}$. If $N(u)-\{v, w\} \subseteq N(v) \cup N(w)$ in $G$ and $i d(u)=$ $\min \{i d(u), i d(v), \overline{i d}(w)\}$, then change the marker of $u$ to $F$.

Again, it is easy to prove that $V^{\prime}-\{u\}$ is still a connected dominating set. However, Property 2 usually does not hold. 

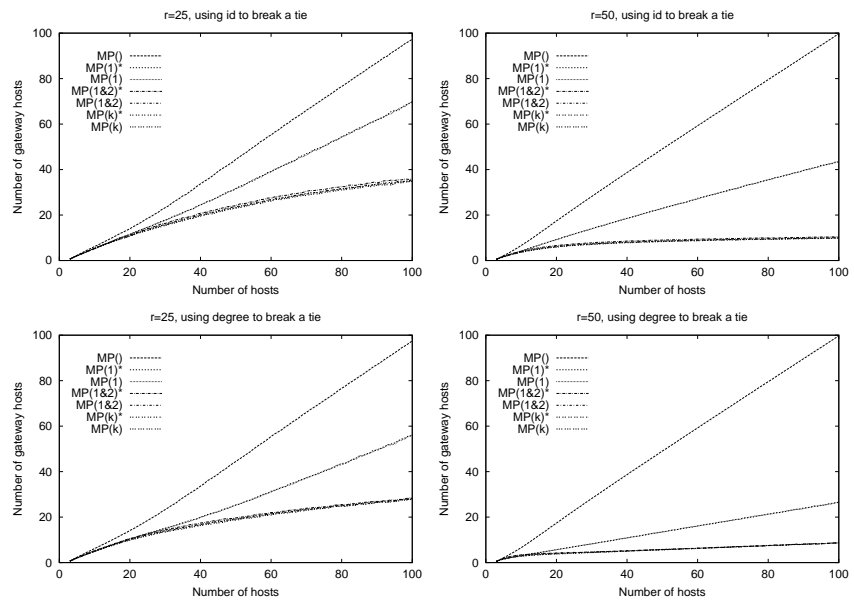

Figure 3. Size of dominating set generated by
different versions of the marking process.

Although $v$ and $w$ are directly connected in Rule 2, they may or may not be neighbors of $u$.

Consider the example in Figure 1 (c) where both $v$ and $w$ are neighbors of $u$. Clearly, $N(u)-\{v, w\} \subseteq N(v) \cup N(w)$. If $i d(u)=\min \{i d(u), i d(v), i d(w)\}$, vertex $u$ can be removed from $V^{\prime}$ based on Rule 2. If $i d(v)<i d(u)$ then vertex $v$ can be removed based on Rule 1 , since $N(v)-\{u\} \subseteq$ $N(u)$. If $i d(w)<i d(u)<i d(v)$ no vertex can be removed. Therefore, the id assignment also decides the final outcome of the dominating set. In the subsequent discussion, we use the alphabetic order of vertex label to order id's. For example, $u<v<w$.

If $u$ and $v$ are neighbors in Rule 1 , Rule 1 is called restricted. Similarly, if $u$ is neighbor of both $v$ and $w$ in Rule 2 , Rule 2 is called restricted. We will see later that it is relatively easy to implement restricted Rule 1 (Rule 2) in a localized way. To apply restricted Rule 1 or Rule 2 , an additional last step in the marking process needs to be included: If $u$ is marked ( $m(u)=T)$, send its status to all its neighbors; i.e., each host needs to keep 2-hop neighborhood information. To apply non-restricted Rule 1 and Rule 2, $u$ 's status needs to be transferred one hop further; i.e., each host needs to keep 3-hop neighborhood information.

Rule 1 and Rule 2 can be easily extended to a more general rule where the neighbor set of vertex $u$ is covered by the union of neighbor sets of more than two vertices in $V^{\prime}$.

Rule $k$ : Assume that $\left\{v_{1}, v_{2}, \ldots, v_{k}\right\}$ is the vertex set of a connected subgraph in $G^{\prime}$. If $N(u)-$ $\left\{v_{1}, v_{2}, \ldots, v_{k}\right\} \subseteq N\left(v_{1}\right) \cup N\left(v_{2}\right) \cup \ldots \cup N\left(v_{k}\right)$ in $G$ and $i d(u)=\min \left\{i d(u), i d\left(v_{1}\right), i d\left(v_{2}\right), \ldots, i d\left(v_{k}\right)\right\}$, then change the marker of $u$ to $F$.

One problem in applying Rule $k$ is its high computation cost, even if the restricted Rule $k$ is applied where the com- putation complexity is choosing $k$ out of $|N(u)|$ neighbors of $u$. Note that other metrics can be used to break a tie; for example, vertex degree (number of neighbors), energy level, and geographical location of vertex in a particular dimension.

Figure 2 shows an ad hoc network generated by the simulation software in a confined space of $100 \times 100$. There are 80 hosts each of which has a transmitter range of 20 . Rule $k$ unmarks only two more gateways than Rules 1 and 2 do. Figure 3 shows simulation results on the average size of dominating set generated by $M P(), M P(1), M P(1)^{*}$, $M P(1 \& 2), M P(1 \& 2)^{*}, M P(k)$, and $M P(k)^{*}$. It is clear from the results that $M P(k)$ does not improve much in reducing the number of gateways compared with $M P(1)$ and $M P(1 \& 2)$, especially in reasonably dense networks. Considering its high computation cost, $M P(k)$ will not be considered in the subsequent discussion.

\section{Dominating Set Update}

Different versions of the marking process. We consider five versions of the marking process: (1) Marking process(MP) without Rule 1 and Rule 2: $M P()$. (2) MP with restricted Rule 1 only: $M P(1)^{*}$. (3) MP with Rule 1 only: $M P(1)$. (4) MP with restricted Rules 1 and 2: $M P(1 \& 2)^{*}$. (5) MP with Rules 1 and 2: $M P(1 \& 2)$. In restricted Rule 1 and/or Rule $2\left(M P(1)^{*}\right.$ and $\left.M P(1 \& 2)^{*}\right)$, it is required that $u$ and $v$ are neighbors in Rule 1 and $v$ and $w$ are neighbors of $u$ in Rule 2. In this case, 2-hop neighborhood information is sufficient in implementing $M P(1)^{*}$ and $M P(1 \& 2)^{*}$. In $M P(1)$ and $M P(1 \& 2), u$ and $v$ are not necessarily neighbors in Rule 1 . Also, $v$ and $w$ are not necessarily neighbors of $u$ in Rule 2. In this way, 3-hop neighborhood information is needed at each host. By default, vertex id is used to break a tie in Rules 1 and 2. If vertex degree is used to break a tie, subscript "deg" is used, such as $M P(1 \& 2)_{d e g}$.

Consider a graph of four vertices, $u, v, w$, and $x$, with four undirected edges $(u, w),(v, w),(u, x)$, and $(v, x)$ as shown in Figure 4 (a). All four vertices are marked using the marking process. Also, $N(u)=N(v)=\{w, x\}$ $(N(w)=N(x)=\{u, v\})$. Using $M P(1)$, one of $u$ and $v$ (also one of $w$ and $x$ ) is unmarked (and such a vertex is called ex-gateway), leaving two marked vertices ( $x$ and $v$ based on Rule 1). Note that ex-gateway hosts are hosts marked by the marking process but unmarked by one of the rules. Using $M P(1)^{*}$, none of the gateways can be unmarked. Figure 4 (b) shows an example of applying $M P(1 \& 2)$. Note that using $M P(1)^{*}$ and $M P(1 \& 2)^{*}$, gateway $u$ cannot be unmarked.

To simplify the discussion, it is assumed that the marking process (together with Rule 1 and/or Rule 2) can be done quickly between two switch-on/off operations, without requiring each host to apply the marking process at the same time. The period between two switch-on/off operations is 

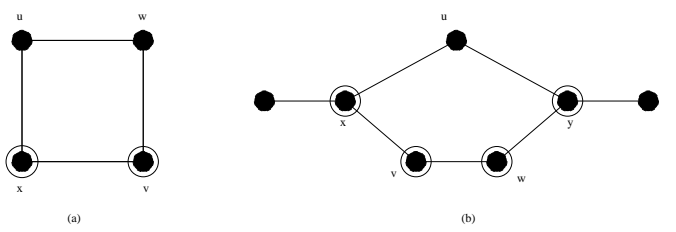

Figure 4. Status after (a) Rule 1, (b) Rule 2.

called a phase. Each host $u$ keeps two statuses: $(m(u)$, $\left.m(u)^{*}\right)$. $m(u)$ stores the result of the marking process. $m(u)^{*}$ stores the final result after applying Rule 1 and/or Rule 2. $m(u)^{*}$ is determined based on $m(v)\left(\right.$ not $\left.m(v)^{*}\right)$ of its neighbor $v \cdot m(u)^{*}$ represents the final status of $u$, and is independent of the sequence in which hosts in the network apply Rule 1 and/or Rule 2.

Update under $M P()$. The marking process has the following desirable locality property: The status of a host (gateway/non-gateway) depends only on connections of its neighbors, not the status of its neighbors. The implication of the locality property is that the status of a host is independent of the status of its neighbors. Therefore, when host $v$ switches on/off, hosts and only hosts that are neighbors of $v$ may change their status.

When a mobile host $v$ switches on, only its non-gateway neighbors, along with host $v$, need to update their status, because any gateway neighbor will still remain as gateway after a new vertex $v$ is added.

Switch-on: (1) Mobile host $v$ broadcasts to its neighbors about its switch-on. (2) Each host $u \in v \cup N(v)$ exchanges its neighbor set $N(u)$ with its neighbors. (3) Host $v$ assigns its marker $m(v)$ to $T$ if there are $(w, v) \in E$ and $\left(v, w^{\prime}\right) \in$ $E$, but $\left(w, w^{\prime}\right) \notin E$. (4) Each non-gateway neighbor $u \in$ $N(v)$ assigns its marker $m(u)$ to $T$ if there is $(w, u) \in E$, but $(w, v) \notin E$.

When a mobile host $v$ switches off, only gateway neighbors of the switch-off host need to update their status, because any non-gateway neighbor will still remain as nongateway after vertex $v$ is deleted.

Switch-off: (1) Mobile host $v$ broadcasts to its neighbors about its switch-off. (2) Each host $u \in N(v)$ exchanges its neighbor set $N(u)$ with its neighbors. (3) Each gateway neighbor $u$ assigns its marker $m(u)$ to $F$ if all neighbors are pairwise connected; that is, $\left(w, w^{\prime}\right) \in E$ for any two neighbors $w$ and $w^{\prime}$ of $u$.

Update under $M P(1 \& 2)^{*}$. When the marking process is used together with Rules 1 and 2, the locality property no longer holds: the status of host $u$ depends also on the status of other hosts ( $v$ in Rule 1 and $v$ and $w$ in Rule 2).

Lemma 1: When the status of host $u$ changes and $u$ is not a neighbor of any switch-on/off host, then it is caused only by using Rule 1 or Rule 2.

Theorem 1: When the dominating set is derived by the marking process with restricted Rules 1 and 2 , and in addition, vertex id is used to break a tie in Rules 1 and 2, hosts and only hosts that are neighbors of switch-on (switch-off) hosts need to update their status.

Proof. Suppose an arbitrarily selected host $u$ is not a neighbor of any switch-on/off host. Based on Lemma 1, $u$ changes its status by neighbor $v(v$ and $w)$ using Rule 1 (Rule 2). Neither $v$ nor $w$ is a switch-on/off host based on restricted Rules 1 and 2 and the way $u$ is selected. The difference between neighbor sets of $v$ and $w$ in the new phase and the previous one is a subset of switch-on/off hosts, with none of them being neighbors of $u$. We consider the following two cases: (1) If host $u$ is changed from non-gateway to gateway, this means that Rule 1 (Rule 2) applied on $u$ in the previous phase cannot be used in the current phase. This occurs when Rule 1 (Rule 2) fails the neighbor coverage condition between $u$ and $v$ in Rule 1 ( $u, v$, and $w$ in Rule 2 ) in the current phase, which is impossible. (2) If host $u$ is changed from gateway to non-gateway, based on neighbor sets of $u$ and $v$ for Rule 1 ( $u, v$ and $w$ for Rule 2), Rule 1 (Rule 2) should have been applied to $u$ in the previous phase. This is a contradiction.

When a mobile host $u$ switches on, only its non-gateway (including ex-gateway) neighbors, along with host $u$, need to update their status by the marking process, while any gateway neighbor will remain as gateway. Specifically, nongateway neighbors may change to gateway neighbors. Using restricted Rules 1 and 2, gateway neighbors may change to ex-gateway neighbors. Ex-gateways in the previous phase that are re-marked by the marking process in the current phase may or may not be unmarked again (back to exgateways) by Rules 1 and 2. When a mobile host $v$ switches off, only gateway neighbors (including ex-gateways) of the host need to update their status by the marking process, while any non-gateway (except ex-gateway) neighbor will still remain as non-gateway after vertex $v$ is deleted. Specifically, gateway neighbors may change their status to the non-gateway status. An ex-gateway neighbor may change back to the gateway status by the marking process, by Rules 1 and 2, or it remains ex-gateway.

Note that when a tie in Rules 1 and 2 is broken by vertex degree instead of vertex id, the locality property no longer holds for restricted Rules 1 and 2. When applying Rules 1 and 2, the host with a smaller vertex degree is changed to ex-gateway. In case of a tie, vertex id is used to break it. Consider the example in Figure 5 (a). When vertex degree is used to break a tie in Rule 1, only host $u$ is gateway and hosts $v, w, x$, and $y$ are ex-gateways. When host $v$ switches off, hosts $u$ and $w$ cover each other with the same vertex degree, $w$ becomes the new gateway ( $u$ becomes ex-gateway) when vertex id is used to break a tie. However, $w$ is not a neighbor of the switch-off host $v$. If vertex id is used to 

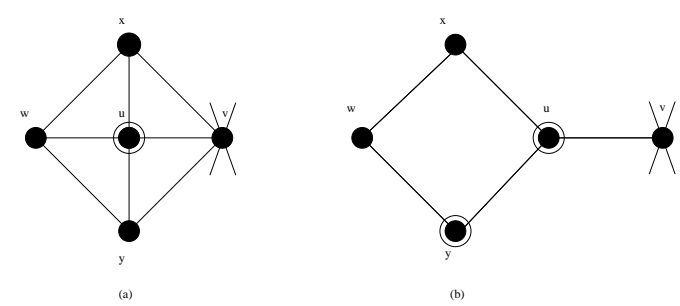

Figure 5 . Violation of the locality property in a switch-off operation (on $v$ ) when vertex degree is used to break a tie.

break a tie in Rules 1 and 2, hosts $u, w$, and $y$ are gateways and host $v$ and $x$ are ex-gateways before host $v$ switches off.

Theorem 2: When the dominating set is derived by the marking process with the restricted Rules 1 and 2, and in addition, vertex degree is used to break a tie, hosts and only hosts that are within 2 hops of switch-on (switch-off) hosts need to update their status.

Proof. Arbitrarily select a host $u$ that is $k$ hops $(k>2)$ away from the switch-on/off host. Based on Lemma 1, the status change of $v$ can only be caused by Rule 1 or Rule 2 . Since Rule 1 (Rule 2) is restricted, other hosts $v(v$ and $w$ ) used in Rule 1 (Rule 2) must be neighbors of $u$; i.e., $(k-$ 1)-hop neighbors of the switch-on/off host. Clearly, vertex degree and vertex id of $v$ and $w$ both remain unchanged in the new phase. Following the similar argument used in the proof of Theorem 1, we conclude that the status of $v$ remains unchanged.

Update under $M P(1 \& 2)$. In $M P(1 \& 2)$, a switch-on/off host may cause the status change of a host that is 2 hops away as shown in Figure 4 (b).

Theorem 3: When the dominating set is derived by the marking process with Rules 1 and 2, and in addition, vertex $i d$ is used to break a tie, hosts and only hosts that are within 2 hops of switch-on (switch-off) hosts need to update their status.

Proof. Suppose an arbitrarily selected host $u$ is not a 1hop or 2-hop neighbor of any switch-on/off host. Based on Lemma 1, $u$ changes its status by neighbor $v(v$ and $w)$ using Rule 1 (Rule 2). Since the neighbor set of $u$ is covered by the neighbor set of $v$ in Rule 1 (and jointly with the neighbor set of $w$ in Rule 2), both $v$ and $w$ are no more than 2 hops away from $u$. Therefore, neither $v$ nor $w$ is a switch-on/off host. The difference between neighbor sets of $v$ and $w$ in the new phase and the old one is a subset of switch-on/off hosts, with none of them being neighbors of $u$. The same arguments used in the proof of Theorem 1 can be applied to show that it is impossible to change the status of host $u$.

In a new phase, in addition to the switch-on (switchoff) procedures, Rules 1 and 2 need to be applied to all
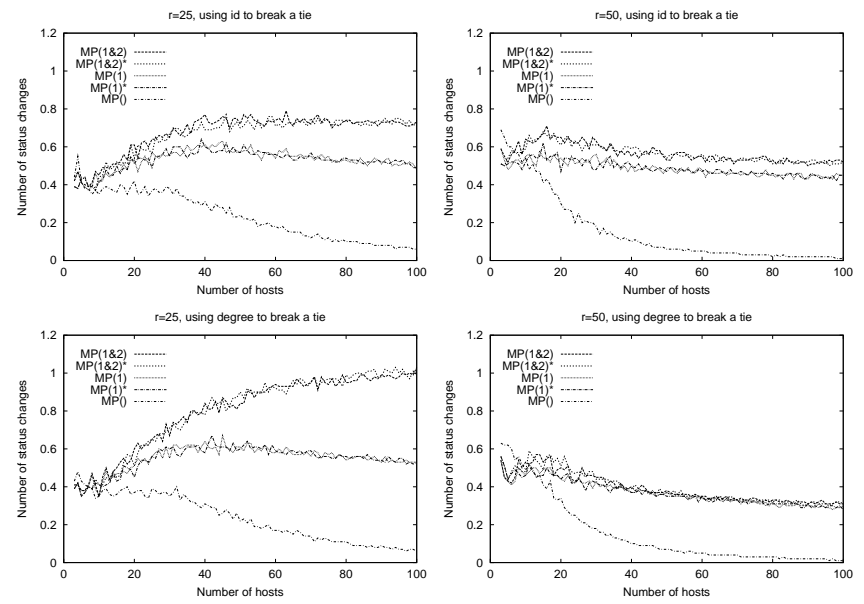

\section{Figure 6. Average number of status changes per switch-on/off.}

switch-on hosts and 1-hop and 2-hop neighbors of switchon (switch-off) hosts.

Theorem 3 fails when vertex degree is used to break a tie. Consider the example in Figure 5 (b) where vertex degree is used to break a tie in Rules 1 and 2. Hosts $u$ and $y$ are gateways, $w$ and $x$ are ex-gateways, and $v$ is non-gateway. When host $v$ switches off, hosts $u$ and $w$ cover each other. Host $u$ becomes ex-gateway and $w$ gateway. However, $w$ is 3 hops away from $v$. On the other hand, the switch-on/off host can only affect the status of neighbors within 3 hops as shown in the following result (its proof is similar to the one for Theorem 3).

Theorem 4: When the dominating set is derived by the marking process with Rules 1 and 2, and in addition, vertex degree is used to break a tie, hosts and only hosts that are within 3 hops of switch-on (switch-off) hosts need to change their status.

All results in this section also apply to $M P(1)^{*}$ and $M P(1)$.

\section{Simulation}

The simulation software generates random connected ad hoc networks within a confined area of $100 \times 100$. Each host in the network is marked as non-gateway, ex-gateway, and gateway by the marking process and the reduction rules. For each network, one random host is added (switch-on) and the status change of other hosts is computed. In the same manner, one random host is removed (switch-off) and the status change is computed. Note that Rules 1 and 2 may be implemented in different ways (restricted or non-restricted, breaking a tie by vertex id or vertex degree). Networks are generated with a fixed transmitter range ( 25 or 50 ), and the 

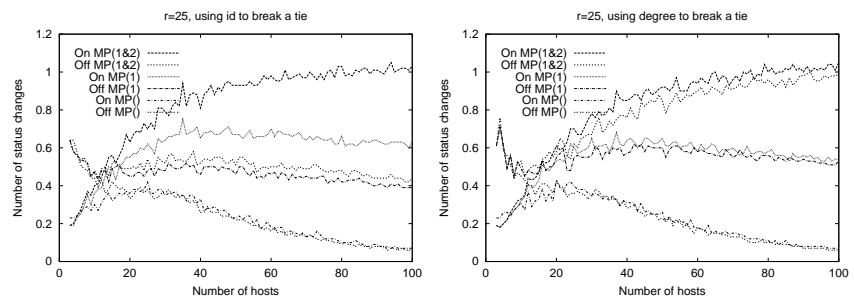

Figure 7. The difference between host switchon and host switch-off.

number of hosts ranges from 3 to 100. All the simulations run for a time long enough to achieve a confidence level of $90 \%$ with a precision within $10 \%$.

Figure 6 shows the number of status changes per switchon/off. Note that when a host switches on (off), only less than one nearby host need to change its status. As to different versions of the marking process, $M P()$ is the most stable and $M P(1 \& 2)$ is the most unstable. The restricted versions $\left(M P(1)^{*}\right.$ and $\left.M P(1 \& 2)^{*}\right)$ and the non-restricted versions $(M P(1)$ and $M P(1 \& 2))$ of Rules 1 and 2 are very close in their stability. However, breaking a tie with vertex id is more stable than with vertex degree, unless when the graph is extremely dense.

Figure 7 compares the difference between host switchon and host switch-off operations. When only $M P()$ is applied or vertex degree is used to break a tie for $M P(1)$ and $M P(1 \& 2)$, the number of status changes caused by switchon and switch-off are very close. However, when vertex id is used to break a tie for $M P(1)$ and $M P(1 \& 2)$, switchoff causes less status changes, thanks to its relatively higher redundancy in the reduced dominating set.

Table 1 shows the average distribution of status changes among the 1-3 hop neighbors of the switch-on/off hosts. The result shows that for all marking processes, any status change can only occur within 1 hop $\left(M P(), M P(1)^{*}\right.$, $\left.M P(1 \& 2)^{*}\right), 2$ hops $\left(M P(1), M P(1 \& 2), M P(1)_{d e g}^{*}\right.$, $\left.M P(1 \& 2)_{d e g}^{*}\right)$, and 3 hops $\left(M P(1)_{d e g}, M P(1 \& 2)_{d e g}\right)$. Note that although $M P(1)$ and $M P(1 \& 2)$ may cause status change 1 hop further than $M P(1)^{*}$ and $M P(1 \& 2)^{*}$, the probability of this kind of status change is very low $(1.98 \%$ when using vertex id and $0.12 \%$ when using vertex degree).

As a conclusion, we can draw the following summary from the simulation results: (1) A host switch-on/off operation only affects the status of its neighborhood within 3 hops $\left(M P(1)_{\text {deg }}, M P(1 \& 2)_{\text {deg }}\right)$. (2) Breaking a tie using vertex id in Rules 1 and 2 is more stable (less status change) than using vertex degree. (3) $M P()$ is more stable than $M P(1)$, which in turn is more stable than $M P(1 \& 2)$. The restricted and non-restricted versions of a reduction method are very close in stability.
Table 1. Locality of marking processes.

\begin{tabular}{|l||r|r|r|r|r|}
\hline Version & Chg\# & 1-hop & 2-hop & 3-hop & Total \\
\hline \hline$M P()$ & 0.23 & $100.00 \%$ & $0.00 \%$ & $0.00 \%$ & $100 \%$ \\
\hline \hline$M P(1)^{*}$ & 0.53 & $100.00 \%$ & $0.00 \%$ & $0.00 \%$ & $100 \%$ \\
\hline$M P(1 \& 2)^{*}$ & 0.66 & $100.00 \%$ & $0.00 \%$ & $0.00 \%$ & $100 \%$ \\
\hline$M P(1)$ & 0.53 & $98.92 \%$ & $1.08 \%$ & $0.00 \%$ & $100 \%$ \\
\hline$M P(1 \& 2)$ & 0.67 & $98.02 \%$ & $1.98 \%$ & $0.00 \%$ & $100 \%$ \\
\hline \hline$M P(1)_{\text {deg }}^{*}$ & 0.58 & $94.89 \%$ & $5.11 \%$ & $0.00 \%$ & $100 \%$ \\
\hline$M P(1 \& 2)_{\text {deg }}^{*}$ & 0.80 & $81.07 \%$ & $19.93 \%$ & $0.00 \%$ & $100 \%$ \\
\hline$M P(1)_{\text {deg }}$ & 0.55 & $93.66 \%$ & $6.33 \%$ & $0.01 \%$ & $100 \%$ \\
\hline$M P(1 \& 2)_{\text {deg }}$ & 0.80 & $78.94 \%$ & $20.94 \%$ & $0.12 \%$ & $100 \%$ \\
\hline
\end{tabular}

\section{Conclusion}

In this paper, we have studied the locality property of the dominating set derived from $\mathrm{Wu}$ and Li's marking process together with several dominating set reduction methods (Rules 1 and 2). Results show that the marking process has good locality property in a system with switch-on/off hosts. Specifically, only 1-hop neighbors of switch-on/off hosts need to update their gateway/non-gateway status when the restricted Rules 1 and 2 is used. 2-hop neighbors of switchon/off hosts need to update their gateway/non-gateway status when the non-restricted Rules 1 and 2 is applied. Our results also show that vertex id is better than vertex degree to break a tie situation in terms of stability of dominating set. All these further confirm that the dominating-set-based routing is a promising approach in ad hoc networks, especially for ones where switch-on/off operations are primary operations that change the network topology.

\section{References}

[1] J. Broch, D. Johnson, and D. Maltz. The dynamic source routing protocol for mobile ad hoc networks. IETF, Internet Draft, draft-ietf-manet-dsr-00.txt, March 1999.

[2] B. N. Clark, C. J. Colbourn, and D. S. Johnson. Unit disk graphs. Discrete Mathematics. 86, 1990, 165-177.

[3] D. Estrin, R. Govindan, J. Heidemann, and S. Kumar. Next century challenges: Scalable coordination in sensor networks. Proc. of ACM MOBICOM'99. 1999, 263-270.

[4] C. E. Perkins. Ad Hoc Networking. Addison Wesley, 2001.

[5] C. E. Perkins and E. M. Royer. Highly dynamic destinationsequenced distance-vector routing (DSDV) for mobile computers. Proc. of ACM SIGCOMM'94. 1994, 234-244.

[6] T. Shepard. A channel access scheme for large dense packet radio networks. Proc. of the SIGCOMM'94 Conference on Communications, Architectures, Protocols, and Applications. 1996.

[7] P. Sinha, R. Sivakumar, and V. Bharghavan. Enhancing ad hoc routing with dynamic virtual infrastructures. Proc. of IEEE INFOCOM. 2001, 1763-1772.

[8] J. Wu and H. Li. On calculating connected dominating sets for efficient routing in ad hoc wireless networks. Proc. of the 3rd Int'l Workshop on Discrete Algorithms and Methods for Mobile Computing and Communications. 1999, 7-14. 